\title{
El femtoscopio puede ser implementado utilizando dispersión de electrones y rayos $\mathrm{X}$ de bajas energías
}

\author{
Edward Henry Jiménez \\ Universidad Central del Ecuador. Facultad de Ingeniería Química \\ ehjimenez@uce.edu.ec \\ Nicolás Recalde Aldunate \\ The University of South Carolina. Physics Department \\ recalde@email.sc.edu \\ Esteban Jiménez Chacón \\ Universite Paul Sabatier. Sciences Technologies et Sante \\ esteban.jimenez@univ-tlse3.fr
}

Recibido: 01 de septiembre / Aprobado: 30 de noviembre 2017

\section{Resumen}

El femtoscopio implica el discernimiento y la diferenciación de cada uno de los elementos presentes en un átomo y molécula, esto implica lógicamente medición de los radios, jamás la posición exacta de protones, neutrones, núcleo y electrones. Este trabajo incrementa el nivel de precisión en la medición atómica y nuclear de los nanómetros, característico del átomo al orden de femtómetros característico de los nucleones. Utilizando los conceptos de resonancia y la ecuación de Schrödinger, se estudia la interacción de electrones y fotones de baja energía con la materia y se mide los radios, atómico y nuclear, para los elementos de la tabla periódica. Se trabajó con 
los datos públicos del NIST y la información de cromatografía de GC/MS de la FIQ-UCE.

En síntesis, la resonancia de rayos $\mathrm{X}$ a bajas energías $(<70 \mathrm{keV})$ da la geometría atómica y nuclear de la capa $\mathrm{K}$. En tanto que la resonancia de electrones a bajas energías $(<2 \mathrm{keV})$ entrega las energías de los electrones de las capas externas y los radios promedios de los átomos.

Palabras clave: femtoscopio, precisión, cromatografía, átomo, radio.

\begin{abstract}
Femtoscope involves the discernment and differentiation of each element in atom and molecule, this logically implies measurement of the radius, never the exact position of protons, neutrons, nucleus and electrons. This work increases the level of precision in the atomic and nuclear measurements of the nanometers, characteristic of the atom to the order of femtometers, characteristic of nucleons.

Using the concepts of resonance and Schrödinger equation, the interaction of electrons and low energy photons with the matter is studied, and the atomic and nuclear radius are measured for elements of periodic table. We worked with the NIST public data and GC/MS chromatography information of the FIQ-UCE.

In synthesis, the resonance of $x$-rays at low energies $(<70 \mathrm{keV})$ gives the atomic and nuclear geometry of the K-layer. Whereas, the resonance of electrons at low energies $(<2 \mathrm{keV})$ gives the energies of outer layers of electron and the average radius of atom.
\end{abstract}

Keywords: femtoscope, precision, chromatography, atom, radio. 


\section{Introducción}

$\mathrm{E}$ xisten pocos estudios de dispersión de electrones y fotones a baja energía. Esto debido a que recientemente se dispone de fuentes de fotones y electrones con alta precisión. A nivel industrial se trabaja de manera cotidiana con GC/ MS, para conocer las concentraciones de una gran variedad de átomos y moléculas. El principio fundamental de la cromatografía de gases y líquidos es la ionización de átomos y moléculas por medio de una fuente de electrones. Las concentraciones de átomos y moléculas medidas en un equipo GC/MS están funcionalmente determinadas por la absorción de la radiación ionizante.

La técnica Low energy electron diffraction LEED, ha sido ampliamente utilizada en el estudio de física de superficies para electrones incidentes con energías de $108 \mathrm{eV}$, dando resultados excelentes [1].

Se ha estudiado la estructura de $\mathrm{MgO}$, con energías de resonancia $530 \mathrm{eV}$. Estos gráficos analizan (Photoemission Intensity $V_{s} \mathrm{E}$ ), no se analiza sección eficaz pero las implicaciones físicas son las mismas [2].

El Laboratorio Nacional de Berkeley [3] presenta un estudio pormenorizado de interacción de rayos X y materia con energías entre 50-30000 eV, donde se presentan las secciones eficaces y los coeficientes de absorción para cada uno de los elementos de la tabla periódica ayuda enormemente al momento de contrastar resultados entre los distintos laboratorios como NIST.

Mediciones de los radios del protón han sido realizados por grupos de investigadores y proyectos, entre ellos MUSE [4][5][6], determinando que se encuentra en un intervalo de (0.83-0.88) fm.

A nivel del electrón no se ha medido de manera directa el radio; existen experimentos que prueban su esfericidad [8].

Mediciones con alto grado de precisión de la sección eficaz de los átomos de la tabla periódica cuando son impactados con rayos X y electrones de baja energía son públicos en la página del NIST, en la cual se menciona de manera fidedigna los autores de los trabajos utilizados como base [9][10][11][12] .

Para que ocurra una reacción de ionización en átomos y moléculas bombardeadas por electrones de baja energía es necesario que mínimo el electrón target y el electrón proyectil se encuentren o interactúen al menos una vez, permitiendo que se concrete una resonancia.

Utilizando un cromatógrafo de gases y un detector de masas, se estudia la dinámica de las reacciones químicas de ionización y su correspondencia con la atenuación másica de la radiación con electrones a bajas energías. Se demuestra la naturaleza probabilista de las reacciones de ionización a través de las ecuaciones de Fisher-Kolmogorov y la formalización clásica [13][14][15][16][17]. Además, utilizando la cinética química se verifica un nuevo método experimental para medir y cuantificar secciones eficaces, correlacionando nuestros resultados con los valores 
del NIST. Finalmente, se testea el algoritmo de cálculo para los elementos presentes en una muestra de aire húmedo. El principal resultado del estudio demuestra que la velocidad de los electrones es el nexo entre la cinética química y la atenuación de radiación a bajas energías: $\mathrm{v}(\mathrm{E})=\mathrm{K}(\mathrm{E}) / \mu(\mathrm{E})=\mathrm{r} / \mathrm{t}$.

Para el caso de fotones y electrones que bombardean materia se demuestra teóricamente que tienen una sección eficaz de resonancia, que concuerda con los modelos teóricos de Teoría de Dispersión [18][19][20].

\section{Materiales y métodos}

\section{Definiciones}

La ecuación fundamental de atenuación de la radiación de $\mathrm{R}_{\mathrm{x}}$, es inversamente proporcional a la distancia r, recorrida por el rayo de la siguiente manera:

$$
I=I_{0} e^{-\mu r}
$$

Cuando un haz de $\mathrm{R}_{\mathrm{x}}$ atraviesa un volumen de espesor $\mathrm{r}$, crea ionización de la materia, y la concentración del número de iones aumenta con el tiempo $\mathrm{C}(\mathrm{t})$.

$$
A+h v \rightarrow A^{+}+e^{-}
$$

De manera similar se produce ionización de átomos y moléculas con un haz de electrones de baja energía $(0,250) \mathrm{eV}$.

$$
A+e^{-} \rightarrow A^{+}+2 e^{-}
$$

Notemos que la frecuencia $v$, corresponde a la frecuencia de ionización de cada elemento de la tabla periódica. En el presente paper trabajaremos normalmente con la longitud de onda $\lambda$, en lugar de la frecuencia, donde la energía y la frecuencia respetan la relación de Planck:

$$
\mathrm{E}=\mathrm{hc} / \lambda=\mathrm{h} v
$$

Dependiendo del tipo de reacción de orden cero, uno o dos, la concentración tendrá la forma: $\mathrm{C}=\mathrm{C}_{0}+\mathrm{Kt},\left(\ln \left(\mathrm{C} / \mathrm{C}_{\mathrm{o}}\right)=\mathrm{K} \mathrm{t}\right)$ y $\left(1 / \mathrm{C}-1 / \mathrm{C}_{0}=\mathrm{K} \mathrm{t}\right)$, donde $\mathrm{K}$ representa la ecuación de Arrhenius.

$$
K=A e^{-\frac{E i}{R T}}
$$


La atenuación de la radiación $\mathrm{I}(\mathrm{x})$ crea átomos ionizados positivamente, si y solamente si su energía es superior a la energía de ionización. Producto de este proceso se crea una concentración de iones $\mathrm{C}(\mathrm{t})$ que aumenta con el tiempo. En síntesis, aumenta la concentración de iones por atenuación de electrones energéticos de manera simétrica, como se expresa en la siguiente ecuación:

$$
\frac{C}{C_{0}}=e^{K t}=\frac{I_{0}}{I}=e^{\mu r}
$$

Debemos considerar que, si solo una fracción $\beta$ de la radiación inicial crea iones, la ecuación queda:

$$
\frac{C}{C_{0}}=e^{K t}=\beta \frac{I_{0}}{I}=\beta e^{\mu r}
$$

De la ecuación (4), obtenemos un resultado fundamental:

$$
K t=\mu r
$$

La ecuación (6), significa que la cinética de una reacción química está definida por la absorción de la radiación, a través de la velocidad de propagación de la radiación ionizante $\mathrm{r}=\mathrm{vt}$. Por lo tanto, la reacción química de la ionización es de orden uno, como se lo expresa en la ecuación (4).

$$
v(E)=\frac{K(E)}{\mu(E)}=\frac{r}{t}
$$

La variable v(E), es la velocidad de la partícula ionizante. Para el caso de electrones esta velocidad está en función del potencial aplicado $\mathrm{V}$, la carga e y masa de electrón $m_{e}$ :

$$
e V=m_{e} \frac{v^{2}}{2}
$$

El lado izquierdo de las ecuaciones (5), (6) representa las funciones que dependen de la variable temporal kt, el lado derecho indica las funciones dependientes del espacio $\mu$ r.

Las ecuaciones (5), (6) y (7) conectan el espacio r y el tiempo t. La interacción de la radiación con la materia depende explícitamente de la variable espacial r; mientras que la cinética de una reacción química depende del tiempo t.

La radiación con electrones y $\mathrm{R}_{\mathrm{x}}$ son la causa de la reacción química y la reacción química es la responsable de la creación de iones hidronio y oxidrilo que cambian la estructura del ADN impidiendo que las moléculas cancerosas sigan reproduciéndose. El radical hidronio es el ácido más fuerte conocido que a su vez destruye células 
cancerosas. Existen efectos colaterales en las células sanas, que a pesar del daño pueden recuperarse, en tanto que las células cancerosas han perdido esa capacidad. Las reacciones químicas fundamentales de este proceso son:

$$
\mathrm{H}_{2} \mathrm{O}+\mathrm{H}_{2} \mathrm{O}+h v \rightarrow \mathrm{H}_{3} \mathrm{O}^{+}+\mathrm{OH}^{-}
$$

La ecuación (8), en forma reducida se escribe como:

$$
\mathrm{H}_{2} \mathrm{O}+h v \rightarrow \mathrm{H}^{+}+\mathrm{OH}^{-}
$$

La atenuación de la radiación está en el dominio del espacio y debidamente descrita por la ecuación (1). La cinética de una reacción química está en el dominio del tiempo debidamente descrita por la evolución de la concentración y por la ecuación de Arrhenius (3). Las dos ecuaciones son equivalentes como se indica en (5), (6) y (7). La variable $\mathrm{C}(\mathrm{t})$, puede medir el valor de la concentración en el tiempo de electrones o iones producidos por la interacción de electrones o rayos $\mathrm{x}$ con la materia.

El átomo se caracteriza por el radio atómico $r$, el número de protones $Z=A-N$, el número de electrones $\mathrm{Z}$ y el número de neutrones $\mathrm{N}$.

\section{Sección eficaz}

Según el NIST, las tabulaciones actuales de $\mu / \rho$ dependen en gran medida de los valores teóricos para la sección transversal total por átomo, $\sigma_{\text {tor }}$, que está relacionado con $\mu$ / $\rho$ mediante la siguiente ecuación:

$$
\frac{\mu}{\rho}=\frac{\sigma_{t o t}}{u A}
$$

En (3), u (= $\left.1.6605402 \times 10^{-24} \mathrm{gr}\right)$ es la masa atómica (1/12 de la masa de 12C) [4].

El coeficiente de atenuación, las secciones transversales de interacción de fotones y las cantidades relacionadas son funciones de la energía del fotón. La sección transversal total se puede escribir como la suma sobre las contribuciones de las interacciones principales de fotones,

$$
\sigma_{\text {tot }}=\sigma_{p e}+\sigma_{c o h}+\sigma_{\text {incoh }}+\sigma_{\text {pair }}+\sigma_{\text {trip }}+\sigma_{p h . n}
$$

Donde $\sigma_{\text {pe }}$ es la sección transversal del efecto fotográfico atómico, $\sigma_{\text {coh }}$ y $\sigma \mathrm{i}_{\text {ncoh }}$ son las secciones transversales de dispersión coherentes (Rayleigh) e incoherentes (Compton), respectivamente, $\sigma_{\text {pair }}$ y $\sigma_{\text {trip }}$ son las secciones transversales para la pro- 
ducción de positrones de electrones en los campos del núcleo y de los electrones atómicos, respectivamente, y $\sigma_{\text {ph.n. }}$ es la sección transversal fotonuclear [3][4].

Utilizamos los datos de NIST para los elementos $Z=11$ a $Z=92$ y las energías de fotones $1.0721 \mathrm{E}-03 \mathrm{MeV}$ a $1.16 \mathrm{E}-01 \mathrm{MeV}$, y se calcularon de acuerdo con:

$$
\frac{\mu}{\rho}=\left(\sigma_{p e}+\sigma_{c o h}+\sigma_{\text {incoh }}+\sigma_{\text {pair }}+\sigma_{\text {trip }}+\sigma_{p h . n}\right) / u A
$$

El coeficiente de atenuación $\mu$ de un rayo de electrones de baja energía $(10,100)$ $\mathrm{eV}$, esencialmente tendrá las componentes elástica e inelástica. Se desprecia Bremsstrahlung emission y Positron annihilation.

$$
\sigma_{\text {tot }}=\sigma_{\text {coh }}+\sigma_{\text {incoh }}
$$

\section{Zona de resonancia para fotones}

Se crea una región de resonancia de forma natural en la capa $\mathrm{K}$ entre el núcleo y los electrones en el nivel S. La condición para que los fotones entren en la región de resonancia viene dada por $r a \geq r_{n}+\lambda$. Esta región de resonancia nos proporciona una nueva forma de entender el efecto fotoeléctrico. Existe evidencia experimental de la existencia de resonancia en el nivel $\mathrm{K}$ debido al efecto fotoeléctrico, representado por la sección transversal de resonancia proporcionada por el NIST para cada átomo. En el presente trabajo nos centramos en los efectos de resonancia, pero no en el origen de la región de resonancia. Teorema 1.

La sección eficaz de resonancia es la responsable de variaciones grandes y/o anómalas en la radiación absorbida $\left(\mathrm{I}_{2}-\mathrm{I}_{1}\right)$, como se lo demuestra en el Teorema 1.

$$
\frac{I_{2}-I_{1}}{\frac{I_{2}+I_{1}}{2}}=-\frac{\rho r}{\mu A}\left(\sigma_{2}-\sigma_{1}\right)
$$

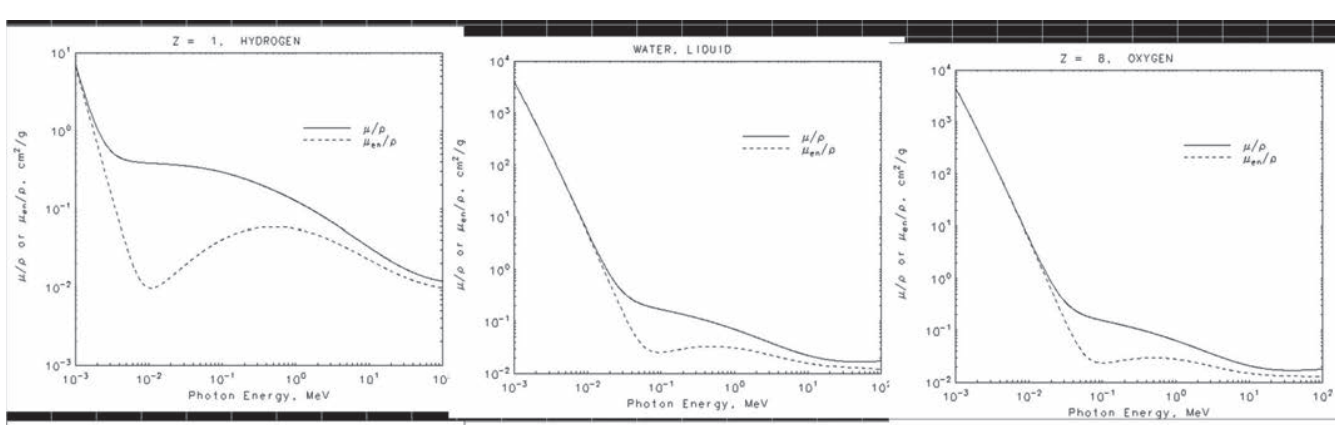



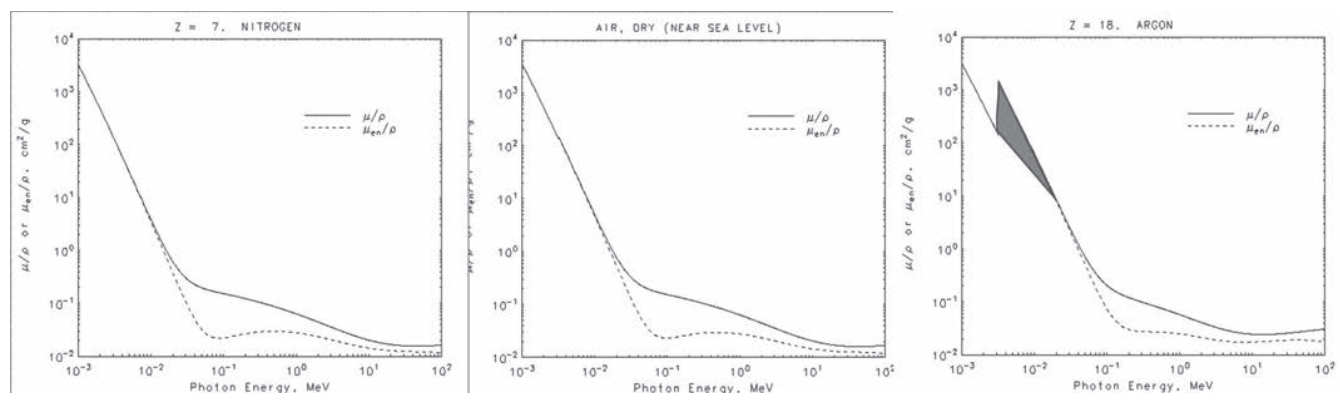

Figura 1. Las figuras anteriores muestran las regiones de resonancia para el argón, pero para los demás son muy pequeñas. La radiación de ionización son rayos X.

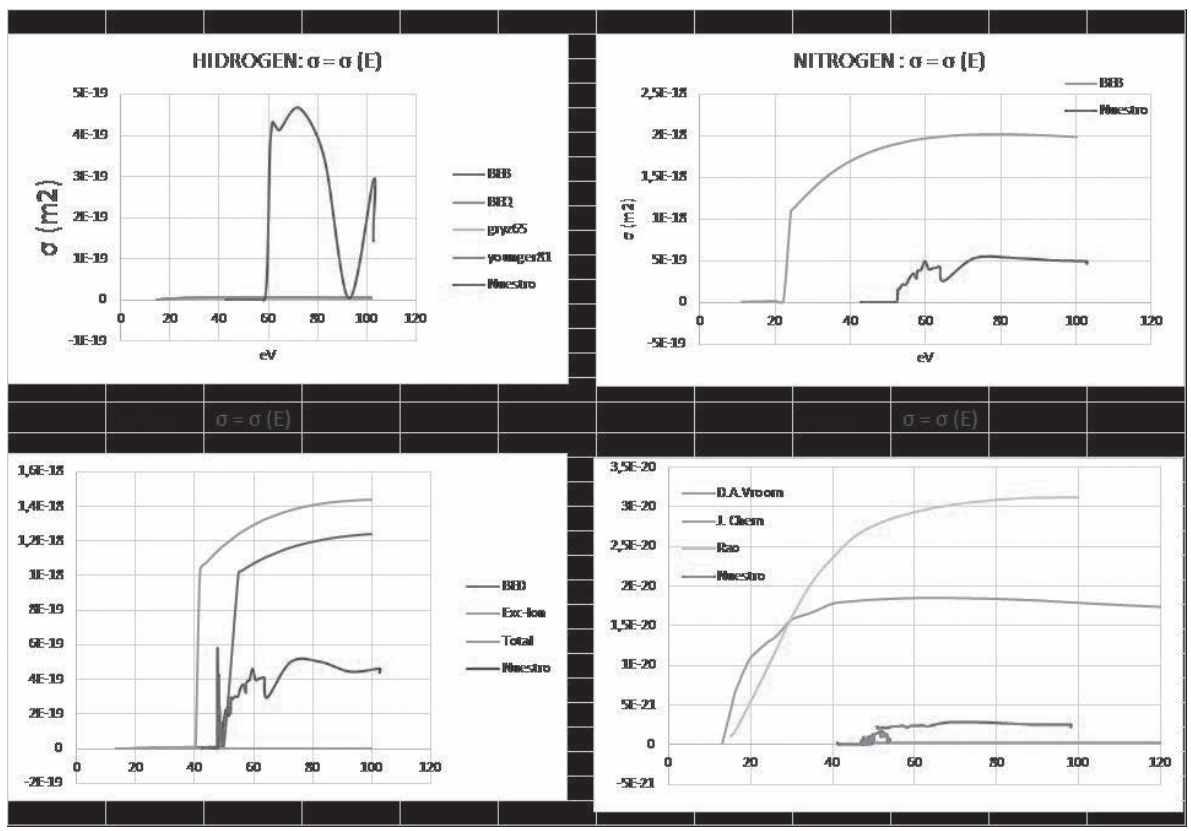

Figura 2. Las figuras anteriores muestran las regiones de resonancia para $\mathrm{H} 2, \mathrm{~N}, \mathrm{O} 2, \mathrm{H} 2 \mathrm{O}$. La radiación de ionización son electrones con energía entre $(0,105) \mathrm{eV}$.

La figura 2 muestra que nuestros datos experimentales concuerdan con la información pública del NIST.

La figura 3 indica claramente que cada átomo y/o molécula tiene una sección eficaz de resonancia representada por el valor máximo de la sección eficaz. Esta sección eficaz máxima tiene un valor único para cada elemento químico y/o compuesto molecular y es la huella digital desde la perspectiva de las bajas energías. Por ejemplo, el radio más probable para el átomo de hidrógeno, representa la longitud de onda de D’Broglie y está dado por $\mathrm{r}_{\mathrm{H}}=\mathrm{h} /(2 \pi \mathrm{m})$. 


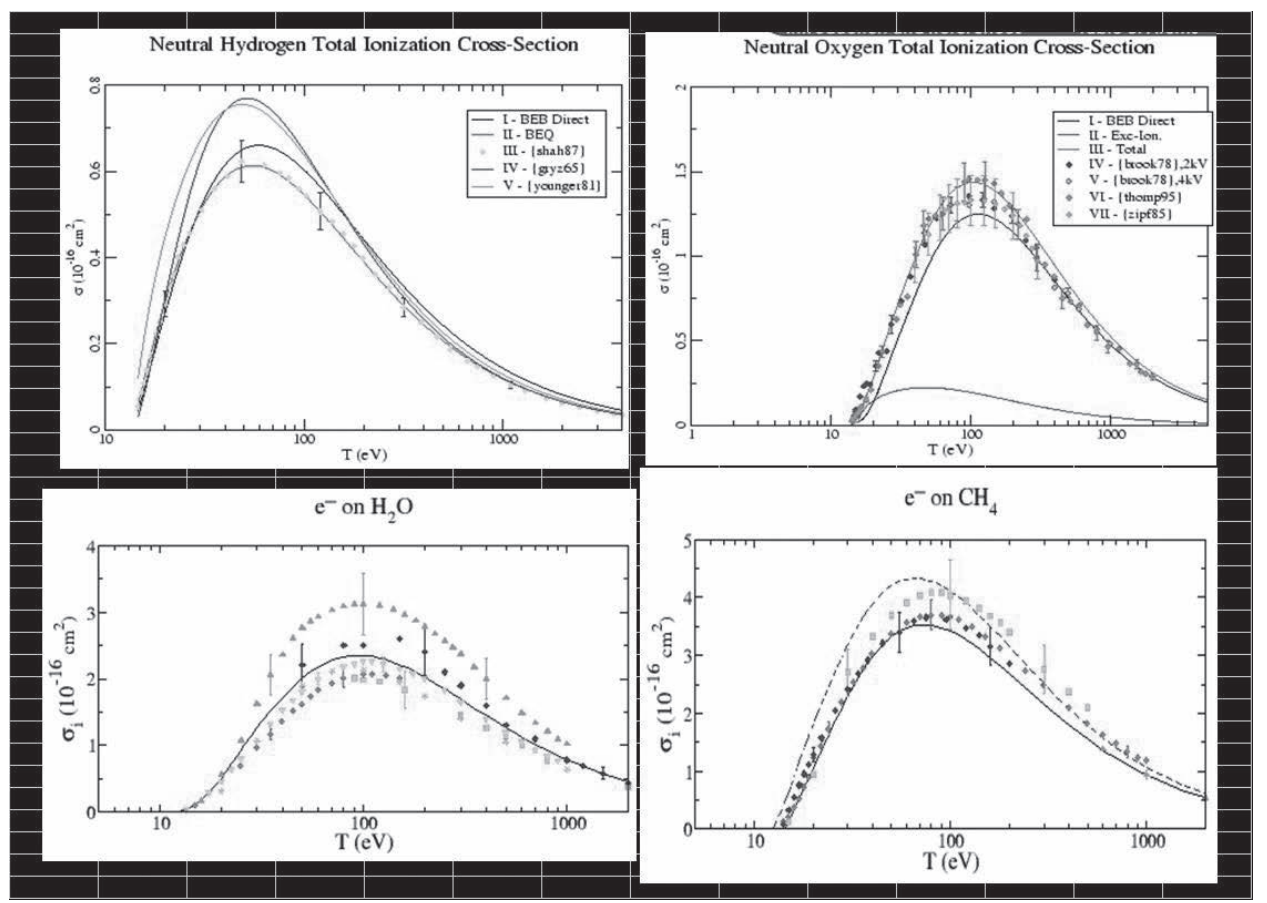

Figura 3. Sección eficaz de algunos átomos y moléculas que indican que existe una resonancia en los valores máximos. Información pública del NIST.

\section{Resultados}

\section{Precisión del modelo de Schrödinger.}

Cuando existe una alta desviación estándar $\langle\Delta \mathrm{r}\rangle=\left(\left\langle\mathrm{r}^{2}\right\rangle-\langle\mathrm{r}\rangle^{2}\right)^{1 / 2}$ en la medición del radio de cualquiera de las órbitas de un átomo es necesario determinar el nivel del error no solo en la posición $€=\langle\Delta \mathrm{r}\rangle \mid\langle\mathrm{r}\rangle$ sino en la energía $€=\langle\Delta \mathrm{E}\rangle \mid\langle\mathrm{E}\rangle$. Esto se lo puede calcular con la aplicación del principio de incertidumbre de Heisenber y los valores promedio de los operadores energía y posición.

En (Leslie Ballentine, p. 295) [18], se presenta los cálculos de error respectivo para la energía, en el estado base del hidrógeno atómico.

$$
\varepsilon=\langle H\rangle_{\min } /\left|E_{100}\right|=-1
$$

En la ecuación anterior se utilizó la función de onda $\psi=\mathrm{Ce}^{-\mathrm{r} / \mathrm{a}}$ obteniéndose $\langle\mathrm{H}\rangle$ $\mathrm{y}\langle\mathrm{H}\rangle_{\text {min }}$ con el método de variaciones y el parámetro a igual al radio de Bohr. 


$$
\langle H\rangle=\frac{\langle\Psi|H| \Psi\rangle}{\langle\Psi \mid \Psi\rangle}=\frac{h^{2}}{8 \pi \mu a^{2}}-\frac{e^{2}}{a}
$$

Utilizando las ecuaciones para los valores promedio del radio r, dadas por Pauling y Wilson en 1935, p. 267 [18].

$$
\begin{aligned}
& \langle r\rangle=\left\langle\Psi_{n, l, m}|r| \Psi_{n, l, m}\right\rangle=n^{2} a\left(1+\frac{1}{2}\left\{1-\frac{l(l+1)}{n^{2}}\right\}\right) \\
& \left\langle r^{2}\right\rangle=\left\langle\Psi_{n, l, m}\left|r^{2}\right| \Psi_{n, l, m}\right\rangle=n^{4} a^{2}\left(1+\frac{3}{2}\left\{1-\frac{l(l+1)-1 / 3}{n^{2}}\right\}\right)
\end{aligned}
$$

Podemos calcular la incertidumbre en el radio del átomo de hidrógeno, en la primera capa $\mathrm{n}=1, \mathrm{l}=0$ :

$$
\langle\Delta r\rangle=\sqrt[2]{\left\langle r^{2}\right\rangle-(\langle r\rangle)^{2}}=a \sqrt{\frac{3}{4}}
$$

Con lo que el nivel de error o incertidumbre queda determinado por:

$$
\epsilon=\frac{\langle\Delta r\rangle}{\langle r\rangle}=57,7 \%
$$

Todo experimento que se haga para la primera capa del átomo de hidrógeno está restringido a este nivel de error o incertidumbre.

¿Se puede determinar el nivel de error o incertidumbre en la determinación del radio y la energía de átomos y moléculas?

La ecuación (14.5), nos da una idea de cómo calcular la incertidumbre de los modelos físico-matemáticos del átomo de Bohr. Sin embargo, podemos aplicar de manera similar esta metodología para átomos y moléculas generales. Simplemente necesitamos encontrar la ecuación de onda de átomos y moléculas. Presentamos una manera nueva en la determinación de la función de onda a partir de las curvas de resonancia.

Reescribamos la ecuación, debidamente demostrada en la ecuación (7) y en los teoremas 1 y 2 .

$$
v(E)=\sqrt[2]{2 E / m}=\frac{K(E)}{\mu(E)}=\frac{<r>}{<t>}=\frac{<\Delta r>}{<\Delta t>}
$$

Donde $\mathrm{E}$, $\mathrm{m}$ son la energía y la masa del electrón respectivamente. Las relaciones de $<\mathrm{r}>|<\mathrm{t}\rangle$ también se cumplen para las desviaciones estándar $\langle\Delta \mathrm{r}\rangle|<\Delta \mathrm{t}\rangle$.

Los teoremas 1 y 2 establecen la forma explícita de la función de probabilidad para una reacción de ionización creciente. Las ecuaciones [6], [7] y [22] permiten escribir la ley de probabilidad logística que representa a la variable aleatoria r que representa el radio del átomo o molécula. 


$$
P(r)=\frac{\sigma}{\sigma_{a}}=\frac{1}{1+e^{\left(\frac{r-r_{a}}{s}\right)}}=\frac{1}{1+e^{\frac{\sigma \rho}{u A}}\left(r-r_{a}\right)}
$$

Donde el parámetro de entropía está representado por $s=\mathrm{uA} / \sigma \rho$. Notemos que la sección eficaz es un punto fijo del sistema. La media de la ley de probabilidad que representa el radio externo del átomo está definida como:

$$
\langle r\rangle=r_{a}=\int_{0}^{+\infty} r \frac{e^{\left(\frac{r-r_{a}}{s}\right)} d r}{s\left(1+e^{\left(\frac{r-r_{a}}{s}\right)}\right)^{2}}=\int_{0}^{+\infty} \Psi r \Psi d r
$$

De donde la función de onda tiene la forma:

$$
\Psi(r)=\sqrt{\frac{e^{\left(\frac{r-r_{a}}{s}\right) d r}}{s\left(1+e^{\left(\frac{r-r_{a}}{s}\right)}\right)^{2}}}
$$

El valor de la desviación estándar es:

$$
\langle\Delta \mathrm{r}\rangle=\left\langle r^{2}\right\rangle-\langle r\rangle^{2}=\frac{\pi}{\sqrt{3}} S
$$

La entropía $(\ln (s)+2)$, para la densidad de probabilidad del proceso de ionización dado por $\mathrm{f}(\mathrm{r})=\mathrm{P}(1-\mathrm{P})=\psi$, es una variable estratégica del proceso y captura toda la información disponible.

Con lo que el valor de la incertidumbre y la exactitud del modelo buscado para los radios del átomo o la molécula tiene la siguiente forma:

$$
\epsilon=\left(\frac{\langle\Delta r\rangle}{\langle r\rangle}\right)=\frac{\frac{\pi}{\sqrt{3}} S}{r_{a}}
$$

Despejando el valor de $\langle\Delta \mathrm{r}>=\mathrm{v}(\mathrm{E})<\Delta \mathrm{t}>\mathrm{y}$ aplicando la relación (14.7) tenemos el valor de $\langle\Delta t>$ que se relaciona con el valor de la energía, de la siguiente manera:

$$
\langle\Delta \mathrm{E}\rangle=\frac{\hbar}{\langle\Delta \mathrm{t}\rangle}=\frac{\hbar v(E)}{\langle\Delta \mathrm{r}\rangle}
$$


El cálculo del valor del parámetro s, puede hacerse con los datos experimentales de la figura 3, donde tenemos la sección eficaz en función de la energía. Pero a su vez la energía está en función de la onda asociada al electrón que representa el radio del átomo $(\lambda=r)$.

$$
\ln \left(\frac{\sigma_{0}-\sigma}{\sigma}\right)=\frac{1}{s}\left(r-r_{a}\right)
$$

Teorema. Región de resonancia. La sección transversal de resonancia se produce por la interferencia entre el núcleo atómico y los rayos $\mathrm{X}$ entrantes dentro de la región de resonancia, donde los límites son la superficie del núcleo atómico y la cubierta K.

La sección eficaz del núcleo atómico está dada por:

$$
\sigma_{r_{n}}=4 \Pi r_{n}^{2}=4 \Pi A^{2 / 3} r_{n}^{2}
$$

La sección transversal del fotón en la concha $\mathrm{K}$ depende de la longitud de la onda y la forma del núcleo atómico:

$$
\sigma_{r_{n}+\lambda}=4 \Pi\left(r_{n}+\lambda\right)^{2}
$$

Restando las secciones transversales (13) y (14) tenemos:

$$
\sigma_{\lambda}=\sigma_{r_{n}+\lambda}-\sigma_{r_{n}}=4 \Pi\left(2 r_{n} \lambda+\lambda^{2}\right)=4 \Pi\left(2 r_{p} \lambda+2\left(r_{n}-r_{p}\right) \lambda+\lambda^{2}\right)
$$

La resonancia se produce por las interacciones entre los rayos X, los electrones K-shell y el núcleo atómico. Las secciones transversales correspondientes al núcleo están ponderadas por la probabilidad $\mathrm{p}_{\mathrm{n}} \mathrm{y}$ deben tener una dependencia simple de un término de interferencia. Este último depende del radio de protones $\mathrm{r}_{\mathrm{p}}$ o de la diferencia entre el núcleo y el radio de protones $\left(r_{n}-r_{p}\right)$ de acuerdo con la siguiente relación:

$$
\begin{gathered}
\frac{p_{n}+p_{e}}{p_{n}}\left(\sigma_{2}-\sigma_{1}\right)=\operatorname{Max}\left(\sigma_{m}-\sigma_{m-1}\right)=4 \pi\left(2 r_{p} \lambda\right) \\
\frac{p_{n}+p_{e}}{p_{n}}\left(\sigma_{2}-\sigma_{1}\right)=\operatorname{Max}\left(\sigma_{m}-\sigma_{m-1}\right)=4 \pi\left(2\left(r_{n}-r_{p}\right) \lambda\right)
\end{gathered}
$$

Observamos que el lado izquierdo de las ecuaciones (18) y (19) debería tener un factor mayor que uno debido a la resonancia. El factor único que mantiene este requisito es $\left(\mathrm{p}_{\mathrm{n}}+\mathrm{p}_{\mathrm{e}}\right) / \mathrm{p}_{\mathrm{n}}$. Donde: 


$$
P_{n}=\frac{\sigma_{n}}{\sigma_{a}}=\frac{1 \cdot 2^{2} A^{2} / 3}{r_{a}^{2}}
$$

La probabilidad de encontrar al menos un electrón dentro del átomo está representada por el radio de Bohr y se define por:

$$
P_{e}=\frac{\sigma_{e}}{\sigma_{a}-\sigma_{n}}=\frac{r_{e}^{2}}{r a_{a}^{2}-1.2^{2} A^{2 / 3}} .
$$

Debido a razones físicas, el término que se debe tomar es $4 \pi\left(2 \mathrm{r}_{\mathrm{p}} \lambda\right)$ porque esto solo es en el límite de la región de resonancia.

La razón por la que el protón de sección transversal debe ser elíptica es porque solo podemos medir un eje de la elipse y los valores experimentales actuales están en el rango de $0.83 \mathrm{fm}$ a $0.88 \mathrm{fm}$.

Cabe señalar que la interacción de los rayos $\mathrm{X}$ es con un protón dado ubicado en el límite del núcleo. Por lo tanto, la versión semi-empírica de la ecuación (18), que ha sido validada utilizando datos experimentales del NIST y las bases teóricas, se da a continuación por:

$$
\frac{\frac{r_{e}{ }^{2}}{r_{a}^{2}-1.2^{2} A^{2 / 3}}+\frac{1.2^{2} A^{2 / 3}}{r_{a}^{2}}}{\frac{1.2^{2} A^{2 / 3}}{r_{a}^{2}} .}\left(\sigma_{2}-\sigma_{1}\right)=8 \Pi r_{p} \lambda
$$

De la ecuación (20) obtenemos el protón de radio, $r_{\mathrm{p}}$.

\section{Metodología}

\section{Radio del electrón, una aplicación del teorema}

Las dimensiones del protón son dinámicas y corresponden a una elipse. El protón se deforma y aumenta con el peso atómico A, debido a la fuerza nuclear. Usando la teoría de la entropía mínima, podemos calcular la dimensión óptima del radio del protón y las condiciones para que el fotón quede atrapado en la región de resonancia correspondiente a la capa K. Una vez en esta región, el fotón interactúa con el electrón o con uno de los nucleones. En este artículo, proporcionamos un método de cálculo para el radio de protones en función de la sección transversal de resonancia. Sin embargo, de manera similar podemos obtener el radio de los electrones ya que los rayos X de baja energía están confinados entre los límites correspondientes a los electrones en la capa $\mathrm{K}$ y los protones en el núcleo. 


$$
\frac{p_{n}+p_{e}}{p_{e}}\left(\sigma_{2}-\sigma_{1}\right)\left(\frac{A}{A_{R}}\right)^{1 / 3}=4 \pi\left(2 r_{e} \lambda\right)
$$

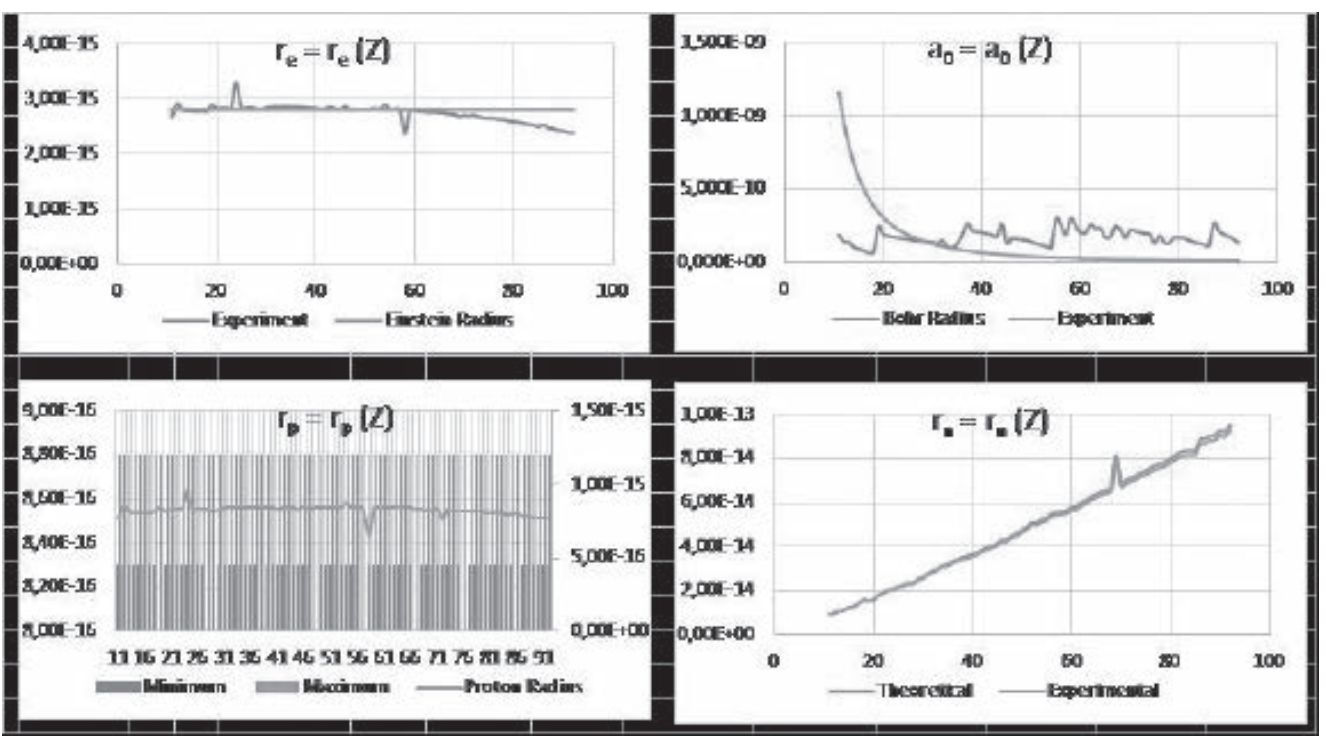

Figura 4. Podemos ver claramente los radios de los electrones, protones, núcleo atómico y radio de Bohr, que demuestran que se puede diferenciar cada uno de los elementos de la tabla periódica en el intervalo $11 \leq \mathrm{Z} \leq 92$.

El femtoscopio podrá discernir claramente los elementos presentes en una muestra.

Para los elementos de $Z \leq 10$, es necesario utilizar en conjunto la información de bajas energías de rayos $\mathrm{X}$ y de electrones.

\section{Conclusiones}

1. De manera radical es totalmente posible diferenciar nucleones en el interior de un átomo con los nucleones en el interior de otro átomo. Este método no puede garantizar nada cuando el protón o el neutrón esta libre. Se podría pensar que en el átomo de hidrógeno el protón es casi libre, sin embargo, no tenemos datos experimentales de resonancia por rayos $\mathrm{X}$ para átomos con $1 \leq \mathrm{Z} \leq 10$. Se necesita completar las tablas de dispersión de rayos $\mathrm{X}$ a bajas energías. Nosotros afirmamos que todos los átomos tienen resonancia en la capa $\mathrm{K}$.

2. Se cumplió el objetivo fundamental del paper, la medición de parámetros atómicos y moleculares utilizando bajas energías. Pues, se midió con gran precisión: los radios promedios del protón y del electrón; el radio promedio de la capa $\mathrm{K}$ y el radio externo promedio para cada uno de los átomos de la tabla periódica. Se calculó el radio del núcleo en función de los radios del protón y el neutrón, con un coeficiente de correlación igual a 1. Además, se demostró a nivel experimental, la convergencia del radio del electrón al radio clásico de Einstein. 
3. El uso de la física tradicional sigue siendo importante en las aplicaciones industriales siempre y cuando los resultados tengan un mínimo error. Cuándo y por qué utilizar electrodinámica en lugar de mecánica cuántica o scaterring theory, seguirán siendo interrogantes en el futuro. Sin embargo, en este trabajo se ha conjugado las dos, en función de los resultados. La resonancia de fotones y materia está muy bien explicada con la interferencia de ondas y el teorema de Mínima Entropía. Mientras que la resonancia de electrones con la materia necesitó de la ecuación de Schrödinger y de la interferencia clásica. Lo aprendido de este paper estuvo guiado por los niveles del error en la medición de parámetros como, por ejemplo: el radio del electrón con un error del 5,47\%, mientras que la medición del radio del protón tuvo un error del $4,14 \%$, que incluso es inferior a las medidas de los mejores laboratorios del mundo.

4. Trabajos de dispersión a bajas energías implica costos de investigación reducidos. Trabajar con bajas energías no solo es beneficioso en costos de equipos y tecnologías, sino que permite encontrar aplicaciones industriales inmediatas. Los equipos de bajas energías para fotones y electrones son equipos de rayos $\mathrm{X}$ y cañones de electrones comerciales y debidamente probados por el sector económico real y productivo. Por ejemplo, para los experimentos con electrones se utilizó un cromatógrafo de gases y un detector de masas GC/MS Agilent, que se utiliza en la industria, medicina y especialmente en refinación de petróleo. El equipo de rayos $\mathrm{X}$, es más fácil con los avances de la electrónica. El estudio presentado en este paper utilizó los resultados públicos del NIST, que concuerdan correctamente con nuestra información, como se lo reporta debidamente en la figura 2 y tabla 1.

5. Experimentos con bajas energías facilitan la integración de la academia con la industria. En nuestros laboratorios de Cromatografía de gases y líquidos, Catálisis, Energías alternativas y Biotecnología tenemos clientes de la industria petrolera, alimentos y farmacéutica, quienes tienen necesidades fundamentales en procesos de refinación de petróleo, huella genética de material cancerígeno en alimentos y tiempo de vida de medicamentos. En este sentido, hemos creado grupos multidisciplinarios que estudian la interacción de rayos $\mathrm{X}$ y electrones a bajas energías con la materia. Estos grupos multidisciplinarios fueron los principales contribuyentes al paper, en especial las ideas de resonancia y tiempos óptimos en los procesos de cinética química.

\section{Referencias}

[1] D. Human et al., Low energy electron diffraction using an electronic delay-line detector, Rev. Sci. Inst. 77023302 (2006).

[2] V. K. Lazarov, R. et al., Structure of the hydrogen-stabilized MgO (111)-(1x1) polar surface: Integrated experimental and theoretical studies, Phys. Rev. B 71, 115434. 2005. 
[3] B. L. Henke et al., X-Ray Interactions: Photoabsorption, Scattering, Transmission, and Reflection at $\mathrm{E}=50-30,000 \mathrm{eV}, \mathrm{Z}=1-92$, Atomic Data and Nuclear Data Tables, Volume 54, Issue 2, July 1993.

[4] Strauch Steffen, Fifth Workshop of the APS Topical Group on Hadronic Physics, April 2013, Denver, CO, USA.

[5] Strauch Steffen, PANIC14, Hamburg, Germany, August 24 - 29, 2014 abstract.

[6] Wong Samuel, Introductory Nuclear Physics, Second Edition, Wiley-VCH Verlag GmbH \& C0. KGaA, Germany, 2004.

[7] R. Pohl et al., The size of the proton, Nature 466, 213 (2010).

[8] J. J. Hudson et al., Improved measurement of the shape of the electron, Nature Letters 473, 2011.

[9] N. Lj. Duric, I. M. Cadez, and M. V. Kurepa, Int. J. Mass. Spectrom. Ion Processes 83, R7 (1988).

[10] J. Schutten, F. J. de Heer, H. R. Moustafa, A. J. H. Boerboom and J. Kistenmaker, J. Chem. Phys. 44, 3924 (1966).

[11] M. A. Bolorizadeh and M. E. Rudd, Phys. Rev. A 33, 882 (1985).

[12] M. V. V. S. Rao, I. Iga and S. K. Srivastava, J. Geophys. Res. 100, 26421 (1995).

[13] S. P. Khare and W. J. Meath, J. Phys. B 20, 2101 (1987), and references therein (theory).

[14] H. C. Straub, B. G. Lindsay, K. A. Smith, and R. F. Stebbings, J. Chem. Phys. 108, 109 (1998).

[15] Atkins P. and de Paula J., Physical Chemistry,W.H. Freeman and Company, New York, 2006, pp. 870-880.

[16] Kolmogorov A, Petrovsky I and Piscounov N. Study of the diffusion equation with growth of the quantity of matter and its application to a biological problem, Bull. State Univ. Mos, (trans. by F. Oliveira- Pinto and B. W). Conolly, Applicable mathematics of non-physical phenomena, Ellis Horwood, 1937, 1982, pp.169-184.

[17] Goldstein H, Poole Ch and Safko J. Classical Mechanics, Addison Wesley, San Francisco, 2001, pp. 483-525.

[18] Leslie Ballentine. Quantum Mechanics. A Modern Development. World Scientific, 1998.

[19] Nakamura H, Mil'nikov G. Quantum Mechanical Tunneling in Chemical Physics. CRC Press Taylor \& Francis Group, Boca Raton, Florida, 2013, pp. 17-19.

[20] Bell R. P, The Tunnel Effect in Chemistry, Springer U.S, 2013, pp. 1-11; 32-62.

[21] H. W. Kuhn and A. W. Tucker, Nonlinear programming, in (J. Neyman, ed.) Proceedings of the Second Berkeley Symposium on Mathematical Statistics and Probability, University of California Press, Berkeley, 1951, pp. 481-492.

[22] L. A. Pars, An Introduction to the Calculus of Variations, John Wiley \& Sons, New York, 1962. 


\section{ANEXOS}

Tabla 1. Continuación. Valores promedios de concentración en el GC/MS para los elementos químicos presentes en una muestra de aire húmedo: $\mathrm{H}, \mathrm{He}, \mathrm{O}, \mathrm{N}, \mathrm{H}, \mathrm{O}, \mathrm{O}, \mathrm{N} . \mathrm{Ar}, \mathrm{Kr}$.

\begin{tabular}{|c|c|c|c|c|c|c|c|c|c|c|}
\hline Voltage & Repeler V & 2 & 4 & 14 & 16 & . & 28 & 32 & 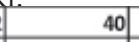 & 83 \\
\hline v & v & $\mathrm{H}_{2}$ & $\mathrm{He}$ & $\mathrm{N}$ & o & $\mathrm{H}_{2} \mathrm{O}$ & $\mathrm{N}_{2}$ & $\mathrm{O}_{2}$ & $\mathrm{Ar}$ & $\mathrm{Kr}$ \\
\hline 10,0 & 31,31 & & & & & & & & & \\
\hline 10,1 & 31,31 & & & & & & & \begin{tabular}{l|l|}
1,27 & \\
\end{tabular} & & \\
\hline 10,2 & 31,31 & & & & & & 1,22 & 2,27 & & \\
\hline 10,3 & 31,31 & & & & & & 4,79 & 4,15 & & \\
\hline 10,4 & 31,31 & & & & & & 6,20 & 4,72 & & \\
\hline 10,5 & 31,31 & & & & & & 8,36 & 5,56 & & \\
\hline 10,6 & 31,31 & & & & & & 9,92 & 5,75 & & \\
\hline 10,7 & 31,31 & & & & & & 13,57 & 6,65 & & \\
\hline 10,8 & 31,31 & & & & & & \begin{tabular}{l|l|}
16,63 \\
\end{tabular} & 7,00 & & \\
\hline 10,9 & 31,31 & & & & & & 18,95 & 6,99 & & \\
\hline 11,0 & 31,31 & & & & & & 24,03 & 9,52 & & \\
\hline 11,1 & 31,31 & & & & & & 28,13 & 9,57 & & \\
\hline 11,2 & 31,31 & & & & & & 35,04 & 9,74 & & \\
\hline 11,3 & 31,31 & & & & & & \begin{tabular}{ll|}
41,46 \\
\end{tabular} & 12,06 & & \\
\hline 11,4 & 31,31 & & & & & & 47,81 & 11,50 & & \\
\hline 11,5 & 31,31 & & & & & & 115,02 & 56,04 & & \\
\hline 11,6 & 31,31 & & & & & & 63,57 & 12,66 & & \\
\hline 11,7 & 31,31 & & & & & & 74,24 & \begin{tabular}{l|l|}
14,27 \\
\end{tabular} & & \\
\hline 11,8 & 31,31 & & & & & & 76,77 & 14,63 & & \\
\hline 11,9 & 31,31 & & & & & & 2,55 & & & \\
\hline 12,0 & 34,5 & & & & & & & & & \\
\hline 12,1 & 34,5 & & & & & & & & & 661,65 \\
\hline 12,2 & 34,5 & & & & & & & & & 685,73 \\
\hline 12,3 & 34,5 & & & & & & & & & 1651,22 \\
\hline 12,4 & 34,5 & & & & & & & & & 907,23 \\
\hline 12,5 & 34,5 & & & & & & & & & 497,02 \\
\hline 12,7 & 34,5 & & & & & & & & & \\
\hline 13,0 & 34,5 & & & & & 11,29 & 1080,99 & 407,29 & \begin{tabular}{l|l|}
10,27 \\
\end{tabular} & 12,68 \\
\hline 13,1 & 34,5 & & & & & & & & & \\
\hline 13,3 & 34,5 & & & & & & & & & \\
\hline 13,4 & 34,5 & & & & & & 101,02 & 37,57 & & \\
\hline 13,5 & 34,5 & & & & & & 164,92 & $\begin{array}{ll}56,15 \\
\end{array}$ & 1,08 & \\
\hline 13,6 & 34,6 & & & & & 1,37 & 409,79 & 140,73 & & 1,43 \\
\hline 13,8 & 34,6 & & & & & & 316,05 & 106,06 & 2,16 & \\
\hline 13,9 & 34,6 & & & & & & 166,50 & \begin{tabular}{l|l}
49,45 \\
\end{tabular} & & \\
\hline 14,0 & 34,6 & & & & & 11,42 & 1055,46 & 381,46 & 9,61 & 8,86 \\
\hline 14,1 & 34,6 & & & & & & 495,16 & 169,58 & 1,30 & 1,11 \\
\hline 14,2 & 34,6 & & & & & 5,19 & 594,00 & 204,16 & 5,01 & 2,37 \\
\hline 14,3 & 34,6 & & & & & & 141,60 & \begin{tabular}{|l|}
46,42 \\
\end{tabular} & & \\
\hline 14,4 & 34,6 & & & & & 6,06 & 649,58 & 226,08 & 6,85 & 1,82 \\
\hline 14,5 & 34,6 & & & & & & 151,01 & 45,00 & & \\
\hline 14,6 & 34,6 & & & & & 10,61 & 994,94 & 334,61 & 7,73 & 2,36 \\
\hline 14,7 & 34,6 & & & & & 13,46 & 1053,71 & 354,84 & 8,89 & 2,21 \\
\hline 14,8 & 34,6 & & & & & 16,56 & 1097,75 & 366,98 & \begin{tabular}{l|l|}
8,99 \\
\end{tabular} & \\
\hline 14,9 & 34,6 & & & & & 14,47 & 1066,03 & \begin{tabular}{|l|}
350,79 \\
\end{tabular} & 8,94 & 1,87 \\
\hline 15,0 & 34,6 & & & & & 9,73 & 958,25 & 301,65 & 8,04 & \\
\hline 15,1 & 34,6 & & & & & & 198,09 & 51,84 & & \\
\hline 15,2 & 34,6 & & & & & & 201,70 & 54,54 & & \\
\hline 15,3 & 34,6 & & & & & & 192,80 & 54,44 & & \\
\hline 15,4 & 34,6 & & & & & 1,83 & 319,56 & 100,42 & 3,71 & \\
\hline 15,5 & 34,6 & & & & & & 234,12 & $\begin{array}{ll}68,75 \\
\end{array}$ & & \\
\hline 15,6 & 31,51 & & & & & & 232,57 & \begin{tabular}{ll|}
64,28 \\
\end{tabular} & 1,04 & \\
\hline 15,7 & 31,51 & & & & & & 163,30 & 4,17 & & \\
\hline 15,8 & 31,51 & & & & & & 149,72 & \begin{tabular}{|l|l|}
43,83 \\
\end{tabular} & & \\
\hline 15,9 & 31,51 & & & & & & 169,33 & \begin{tabular}{ll|}
44,81 \\
\end{tabular} & & \\
\hline 16,0 & 31,51 & & & & & & 228,15 & \begin{tabular}{ll|}
63,56 & \\
\end{tabular} & 1,49 & \\
\hline
\end{tabular}




\begin{tabular}{|c|c|c|c|c|c|c|c|c|c|c|}
\hline Voltage & Repeler V & 2 & 4 & 14 & 16 & 18 & 28 & 32 & 40 & 83 \\
\hline v & v & $\mathrm{H}_{2}$ & $\mathrm{He}$ & $\mathrm{N}$ & o & $\mathrm{H}_{2} \mathrm{O}$ & $\mathrm{N}_{2}$ & $\mathrm{O}_{2}$ & Ar & $\mathrm{Kr}$ \\
\hline 16,1 & 31,51 & & & & & & 756,12 & 202,06 & 3,57 & \\
\hline 16,2 & 31,51 & & & & & & 137,26 & 40,20 & & \\
\hline 16,3 & 31,51 & & & & & 1,66 & 502,68 & 150,14 & 4,60 & \\
\hline 16,4 & 31,51 & & & & 1,14 & 3,42 & 474,39 & 149,04 & 5,88 & \\
\hline 16,5 & 31,51 & & & & 1,12 & 4,54 & 449,03 & 138,38 & 6,56 & \\
\hline 16,6 & 31,51 & & & & 1,20 & & 196,69 & 47,07 & 1,05 & \\
\hline 16,7 & 31,51 & & & & 2,20 & 5,43 & 626,39 & 185,59 & 6,93 & \\
\hline 16,8 & 31,51 & & & & 1,52 & & 213,56 & 44,00 & & \\
\hline 16,9 & 31,51 & & & & 2,41 & 1,90 & 340,74 & 88,76 & & \\
\hline 17,0 & 31,51 & & & & 4,00 & 14,55 & 1239,65 & 360,21 & 10,63 & \\
\hline 17,1 & 34,6 & & & & 3,90 & 161,64 & 1499,94 & 445,16 & 14,35 & \\
\hline 17,2 & 34,6 & & & & 3,45 & 35,19 & 1484,14 & 437,87 & 13,58 & \\
\hline 17,3 & 34,6 & & & & 4,32 & 21,50 & 1506,52 & 439,31 & 13,71 & \\
\hline 17,4 & 34,6 & & & & 5,80 & 27,70 & 1616,18 & 471,01 & 16,11 & \\
\hline 17,5 & 34,6 & & 1,21 & & 6,42 & 27,88 & 1622,56 & 470,26 & 16,01 & \\
\hline 17,6 & 34,6 & & 3,01 & & 6,87 & 28,23 & 1615,98 & 466,36 & 15,69 & \\
\hline 17,7 & 34,6 & & 7,06 & & 7,43 & 27,14 & 1582,78 & 454,75 & 14,85 & \\
\hline 17,8 & 34,6 & & 14,09 & & 8,53 & 25,14 & 1589,88 & 451,04 & 14,92 & \\
\hline 17,9 & 34,6 & & 22,96 & & 9,71 & \begin{tabular}{|l|}
30,23 \\
\end{tabular} & 1630,22 & 460,22 & 15,47 & \\
\hline 18,0 & 34,6 & & 43,42 & & 10,54 & 30,17 & 1650,24 & 465,11 & 15,40 & \\
\hline 18,1 & 34,6 & & 67,96 & & 11,02 & 28,40 & 1632,11 & 458,80 & 15,50 & \\
\hline 18,2 & 34,6 & & 99,76 & & 11,53 & 28,58 & 1668,50 & 467,51 & 15,61 & \\
\hline 18,3 & 34,6 & & 131,62 & & 11,23 & 33,00 & 1671,24 & 465,63 & 15,04 & \\
\hline 18,4 & 34,6 & & 166,69 & & 11,94 & 38,88 & 1763,18 & 487,98 & 16,47 & \\
\hline 18,5 & 34,6 & & 196,31 & & 13,91 & 43,09 & 1824,79 & 503,16 & 17,63 & \\
\hline 18,6 & 34,6 & & 227,44 & & 14,51 & 54,44 & 1948,71 & 531,24 & 19,18 & \\
\hline 18,8 & 34,6 & & 269,39 & & 15,58 & 73,20 & 2133,69 & 573,85 & 20,87 & \\
\hline 18,9 & 34,6 & & 277,07 & & 9,03 & 2,49 & 1212,66 & 320,69 & 8,29 & \\
\hline 19,0 & 34,6 & & 300,11 & & 9,78 & 2,97 & 1225,09 & 323,10 & 8,54 & \\
\hline 19,1 & 34,6 & & 322,62 & & 9,80 & 3,09 & 1244,12 & 327,36 & 8,59 & \\
\hline 19,2 & 34,6 & & 341,49 & & 11,83 & 3,30 & 1289,52 & 339,41 & 9,54 & \\
\hline 19,3 & 34,6 & & 365,19 & & 12,54 & 3,99 & 1321,44 & 348,78 & 10,05 & \\
\hline 19,4 & 34,6 & & 387,02 & & 12,83 & 5,41 & 1340,70 & 352,58 & 10,23 & \\
\hline 19,5 & 34,6 & & 412,06 & & 12,53 & 6,50 & 1339,68 & 351,51 & 9,08 & \\
\hline 19,6 & 34,6 & & 435,00 & & 12,96 & 6,81 & 1356,55 & 353,24 & 9,92 & \\
\hline 19,7 & 31,3 & & 1468,75 & 1,87 & 43,15 & 1841,13 & 9463,64 & 2251,18 & 265,84 & \\
\hline 19,8 & 31,3 & & 1524,51 & 7,21 & 41,88 & 760,78 & 10143,35 & 2469,87 & 300,37 & 65,04 \\
\hline 19,9 & 31,3 & & 1553,64 & 8,06 & 45,02 & 685,91 & 10862,88 & 2664,07 & 352,65 & 110,23 \\
\hline 20,0 & 31,3 & & 1584,27 & 7,01 & 40,55 & 704,81 & 10597,80 & 2598,64 & 324,39 & 73,82 \\
\hline 21 & 31,3 & & 2373,90 & 17,98 & 54,64 & 1155,82 & 13521,27 & 3203,86 & 499,32 & 157,72 \\
\hline 22,0 & 31,3 & & 2313,36 & 18,87 & 55,94 & 914,51 & 13439,78 & 3194,07 & 472,31 & 125,25 \\
\hline 23 & 31,3 & & 4512,73 & 65,48 & 115,09 & 1580,34 & 21247,01 & 4780,29 & 672,54 & 109,05 \\
\hline 24,0 & 31,3 & & 5676,09 & 127,71 & 165,50 & 1788,84 & 24220,49 & 5385,70 & 701,75 & 85,30 \\
\hline 24,6 & 31,3 & & 20967,93 & 62,22 & 94,51 & 1854,84 & 20568,98 & 4451,96 & 425,16 & \\
\hline 24,7 & 31,3 & & 20272,26 & 64,88 & 95,23 & 1785,57 & 20392,93 & 4404,05 & 418,25 & \\
\hline 24,8 & 31,3 & & 19596,78 & 64,89 & 95,34 & 1701,49 & 19990,42 & 4295,61 & 406,58 & \\
\hline 25,0 & 31,3 & & 7793,68 & 244,74 & 240,56 & 2084,71 & 28529,53 & 6280,86 & 733,65 & 47,65 \\
\hline 26 & 31,3 & & 10804,88 & 333,19 & 306,70 & 2475,82 & 32976,58 & 7203,62 & 825,13 & 48,53 \\
\hline 27,0 & 31,3 & & 34714,83 & 1598,82 & 806,98 & 1234,64 & 46237,71 & 13592,58 & 1442,16 & 156,97 \\
\hline 28 & 31,3 & 50,87 & 97550,31 & 418,82 & 343,61 & 2464,85 & 32109,33 & 6796,59 & 681,16 & 5,80 \\
\hline 29,0 & 31,3 & 114,34 & 118739,35 & 509,25 & 394,83 & 2396,89 & 32693,05 & 6889,24 & 695,39 & 3,16 \\
\hline 30 & 31,3 & 111,46 & 123636,18 & 610,66 & 445,46 & 2520,77 & 34515,87 & 7242,46 & 730,89 & 5,46 \\
\hline 31,0 & 31,3 & 104,85 & 121106,95 & 703,68 & 486,41 & 2653,05 & 36509,87 & 7626,78 & 762,43 & 5,01 \\
\hline 32 & 31,3 & & 20914,25 & 56,72 & 90,33 & 1863,79 & 20202,68 & 4383,73 & 421,30 & \\
\hline 40,0 & 28,33 & 202,85 & 721125,32 & 4407,37 & 2267,01 & 10192,74 & 160318,27 & 35924,67 & 3369,60 & 1,08 \\
\hline 50 & 28,33 & 53,95 & 416178,55 & 5284,32 & 2408,91 & 9147,42 & 183267,37 & 44863,35 & 3939,52 & 3,22 \\
\hline 60,0 & 28,33 & & 98899,74 & 3538,98 & 1050,37 & 4058,44 & 120925,67 & 33217,59 & 2976,49 & 2,57 \\
\hline 70 & 28,33 & 29,93 & 138090,70 & 3058,56 & 1490,12 & 3940,39 & 101648,40 & 29120,42 & 2591,19 & 15,69 \\
\hline 70 & 28,33 & 8,05 & 77855,44 & 2345,77 & 1210,22 & 1967,60 & 72857,89 & 21105,09 & 1917,73 & 14,96 \\
\hline 70 & 28,33 & 5,78 & 76352,21 & 2483,90 & 1211,43 & 1692,30 & 69692,96 & 20330,56 & 1861,91 & 12,57 \\
\hline 70 & 28,33 & 5,57 & 108333,95 & 3066,40 & 1504,93 & 2828,68 & 94967,70 & 27582,28 & 2512,84 & 13,98 \\
\hline 70 & 28,33 & 9,10 & 94996,94 & 2838,05 & 1401,85 & 2228,28 & 81813,79 & 23904,26 & 2197,58 & 11,84 \\
\hline
\end{tabular}


Tabla 2. Máxima variación de la sección eficaz a energías específicas o de resonancia, para los elementos de la tabla periódica con: $10<\mathrm{Z}<93$

\begin{tabular}{|c|c|c|c|c|c|c|c|c|}
\hline ELEMENT & $z$ & $\mathbf{N}$ & A & $E_{2}=E_{1}=E$ & $\sigma_{2}$ & $\sigma_{1}$ & $A$ & $(\sigma 2-\sigma 1) / \sigma 1$ \\
\hline & & & A & $\mathrm{MeV}$ & $\mathrm{cm}^{2}$ & $\mathrm{~cm}^{2}$ & $\mathbf{m}$ & $\%$ \\
\hline $\mathrm{Na}$ & 11 & 12 & 23,0 & $1,072 \mathrm{E}-03$ & $2,46 \mathrm{E}-19$ & $2,07 \mathrm{E}-20$ & $1,1580 \mathrm{E}-09$ & $1085 \%$ \\
\hline $\mathrm{Mg}$ & 12 & 12 & 243 & \begin{tabular}{|l|}
$1,305 \mathrm{E}-03$ \\
\end{tabular} & $2.20 \mathrm{E}-19$ & $1,83 \mathrm{E}-20$ & $9.5135 \mathrm{E}-10$ & $1102 \%$ \\
\hline N & 13 & 14 & 27,0 & $1,560 \mathrm{E}-03$ & $1,77 \mathrm{E}-19$ & $1,62 \mathrm{E}-20$ & $7,9605 E-10$ & $993 \%$ \\
\hline Si & 14 & 14 & 28,1 & $1,840 \mathrm{E}-03$ & $1.49 \mathrm{E}-19$ & $1,44 \mathrm{E}-20$ & $6,7474 \mathrm{E}-10$ & $932 \%$ \\
\hline $\bar{p}$ & 15 & 16 & 310 & $2,150 \mathrm{E}-03$ & $1.27 \mathrm{E}-19$ & $1,28 \mathrm{E}-20$ & $5,7745 \mathrm{E}-10$ & $892 \%$ \\
\hline $\mathrm{s}$ & 16 & 16 & 32,1 & $2,470 \mathrm{E}-03$ & $1,10 \mathrm{E}-19$ & $1,16 \mathrm{E}-20$ & $5,0264 \mathrm{E}-10$ & $854 \%$ \\
\hline $\mathrm{Cl}$ & 17 & 18 & 35,5 & $2,822 \mathrm{E}-03$ & $9.65 \mathrm{E}-20$ & $1,04 \mathrm{E}-20$ & $4,3994 \mathrm{E}-10$ & $827 \%$ \\
\hline A & 18 & 18 & 39,9 & $3,203 \mathrm{E}-03$ & $8,46 \mathrm{E}-20$ & $9,47 \mathrm{E}-21$ & $3,8762 \mathrm{E}-10$ & $793 \%$ \\
\hline $\mathrm{K}$ & 19 & 20 & 39,1 & $3,607 \mathrm{E}-03$ & $7,79 \mathrm{E}-20$ & $8,64 \mathrm{E}-21$ & $3,4416 \mathrm{E}-10$ & $802 \%$ \\
\hline $\mathrm{Ca}$ & 20 & 20 & 40,1 & $4,038 \mathrm{E}-03$ & $6.81 \mathrm{E}-20$ & $7,85 \mathrm{E}-21$ & $3,0745 \mathrm{E}=10$ & $767 \%$ \\
\hline Sc & 21 & 24 & 45,0 & $4,490 \mathrm{E}-03$ & $6,08 \mathrm{E}-20$ & $7,23 \mathrm{E}-21$ & $2,7651 \mathrm{E}-10$ & $741 \%$ \\
\hline$\pi$ & 22 & 26 & 47,8 & \begin{tabular}{|l|}
$4,970 \mathrm{E}-03$ \\
\end{tabular} & $5,47 \mathrm{E}-20$ & $6,66 \mathrm{E}-21$ & $2,4980 \mathrm{E}-10$ & $721 \%$ \\
\hline $\mathrm{V}$ & 23 & 28 & 50,9 & $5,465 \mathrm{E}-03$ & $4,97 \mathrm{E}-20$ & $6,16 \mathrm{E}-21$ & $2,2717 \mathrm{E}-10$ & $707 \%$ \\
\hline $\mathrm{Cr}$ & 24 & 28 & 52,0 & $5,989 \mathrm{E}-03$ & $5,16 \mathrm{E}-20$ & $5,68 \mathrm{E}-21$ & $2,0729 \mathrm{E}-10$ & $809 \%$ \\
\hline Mn & 25 & 30 & 54,9 & $6,540 \mathrm{E}-03$ & $4,12 \mathrm{E}-20$ & \begin{tabular}{|l|}
$5,29 \mathrm{E}-21$ \\
\end{tabular} & $1,8983 \mathrm{E}-10$ & $679 \%$ \\
\hline $\mathrm{Fe}$ & 26 & 30 & 55,9 & \begin{tabular}{|l|l|}
$7,110 E-03$ \\
\end{tabular} & $3.78 \mathrm{E}-20$ & $4,93 \mathrm{E}-21$ & $1,7462 \mathrm{E}-10$ & $667 \%$ \\
\hline Co & 27 & 32 & 58,9 & $7,710 \mathrm{E}-03$ & $3,48 \mathrm{E}-20$ & $4,61 \mathrm{E}-21$ & $1,6103 \mathrm{E}-10$ & $656 \%$ \\
\hline $\mathrm{Ni}$ & 28 & 30 & 58,7 & $8,330 \mathrm{E}-03$ & $3.21 \mathrm{E}-20$ & \begin{tabular}{|l|}
$4,32 E-21$ \\
\end{tabular} & $1,4904 \mathrm{E}-10$ & $643 \%$ \\
\hline $\mathrm{Cu}$ & 29 & 34 & 63,6 & $8,979 \mathrm{E}-03$ & $2,93 \mathrm{E}-20$ & $4,04 \mathrm{E}-21$ & $1,3827 \mathrm{E}-10$ & $626 \%$ \\
\hline $\mathrm{Zn}$ & 30 & 34 & 65,4 & $9,660 \mathrm{E}-03$ & $2,76 \mathrm{E}-20$ & $3,81 \mathrm{E}-21$ & $1,2852 E-10$ & $624 \%$ \\
\hline Ga & 31 & 38 & 69,7 & $1,037 \mathrm{E}-02$ & $2,56 \mathrm{E}-20$ & \begin{tabular}{|l|}
$3,59 \mathrm{E}-21$ \\
\end{tabular} & $1,1976 \mathrm{E}-10$ & $613 \%$ \\
\hline Ge & 32 & 41 & 72,6 & $1,110 \mathrm{E}-02$ & $2,39 \mathrm{E}-20$ & $3,39 \mathrm{E}-21$ & $1,1185 \mathrm{E}-10$ & $605 \%$ \\
\hline As & 33 & 42 & 74,9 & $1,187 \mathrm{E}-02$ & $223 \mathrm{E}-20$ & $3,21 \mathrm{E}-21$ & $1,0462 \mathrm{E}-10$ & $595 \%$ \\
\hline $\mathrm{Se}$ & 34 & 44 & 79,0 & $1,266 \mathrm{E}-02$ & $2,08 E-20$ & $3,04 \mathrm{E}-21$ & $9,8083 \mathrm{E}-11$ & $586 \%$ \\
\hline $\mathrm{Br}$ & 35 & 44 & 79,9 & $1,347 \mathrm{E}-02$ & $1,95 \mathrm{E}-20$ & \begin{tabular}{|l|}
$2,89 \mathrm{E}-21$ \\
\end{tabular} & $9,2144 \mathrm{E}-11$ & $576 \%$ \\
\hline $\mathrm{Kr}$ & 36 & 48 & 83,8 & $1,433 \mathrm{E}-02$ & $1,83 \mathrm{E}-20$ & $2,74 E-21$ & $8,6664 \mathrm{E}-11$ & $566 \%$ \\
\hline $\mathrm{Rb}$ & 37 & 48 & 85,5 & $1,520 \mathrm{E}-02$ & $1,72 \mathrm{E}-20$ & \begin{tabular}{|l|}
$2,61 \mathrm{E}-21$ \\
\end{tabular} & $8,1684 \mathrm{E}-11$ & $558 \%$ \\
\hline Sr & 38 & 50 & 87,6 & $1,610 \mathrm{E}-02$ & $1,61 \mathrm{E}-20$ & $2,49 \mathrm{E}-21$ & $7,7091 \mathrm{E}-11$ & $546 \%$ \\
\hline$Y$ & 39 & 50 & 88.9 & $1.704 \mathrm{E}-02$ & $1,52 \mathrm{E}-20$ & $2,38 \mathrm{E}-21$ & $7.2866 \mathrm{E}-11$ & $538 \%$ \\
\hline $\mathrm{Zr}$ & 40 & 51 & 91,2 & $1,800 \mathrm{E}-02$ & $1,43 \mathrm{E}-20$ & $2,27 \mathrm{E}-21$ & $6,8985 E-11$ & $531 \%$ \\
\hline $\mathrm{Nb}$ & 41 & 52 & 92,9 & $1,899 \mathrm{E}-02$ & $1,36 \mathrm{E}-20$ & $2,17 \mathrm{E}-21$ & $6,5392 \mathrm{E}-11$ & $523 \%$ \\
\hline $\mathrm{Mb}$ & 42 & 54 & 95,9 & $2,000 \mathrm{E}-02$ & $1,2 \mathrm{BE} \cdot 2 \mathrm{O}$ & $2,09 \mathrm{E}-21$ & $6,2079 \mathrm{E}-11$ & $515 \%$ \\
\hline TC & 43 & 55 & 98,9 & $2,104 \mathrm{E}-02$ & $1,23 \mathrm{E} \cdot 20$ & \begin{tabular}{|l|}
$2,02 \mathrm{E}-21$ \\
\end{tabular} & $5,8996 \mathrm{E}-11$ & $509 \%$ \\
\hline Ru & 44 & 57 & 101,1 & $2,210 \mathrm{E}-02$ & $1,15 E-20$ & $1,91 \mathrm{E} \cdot 21$ & $5,6177 \mathrm{E}-11$ & $504 \%$ \\
\hline $\mathrm{Rh}$ & 45 & 58 & 102,9 & $2,320 \mathrm{E}-02$ & $1,10 \mathrm{E}-20$ & $1,85 E-21$ & $5,3514 \mathrm{E}-11$ & $494 \%$ \\
\hline $\mathrm{Pd}$ & 46 & 60 & 107,9 & $2,440 \mathrm{E}-02$ & $1,06 \mathrm{E} \cdot 20$ & $1,79 \mathrm{E}-21$ & $5,0882 E-11$ & $490 \%$ \\
\hline Ag & 47 & 60 & 107,9 & $2,55 \mathrm{E}-02$ & $9.92 \mathrm{E}-21$ & $1,72 \mathrm{E}-21$ & $4.8660 \mathrm{E}-11$ & $478 \%$ \\
\hline Cd & 48 & 64 & 112,4 & $2,670 \mathrm{E}-02$ & $9,45 \mathrm{E} \cdot 21$ & $1,64 \mathrm{E}-21$ & $4,6499 \mathrm{E}-11$ & $475 \%$ \\
\hline In & 49 & 64 & 114,8 & $2,790 \mathrm{E}-02$ & $9,02 \mathrm{E} \cdot 21$ & $1,59 \mathrm{E}-21$ & $4,4499 \mathrm{E}-11$ & $469 \%$ \\
\hline Sn & 50 & 69 & 118,7 & $2,920 E-02$ & B, $59 \mathrm{E}-21$ & $1,53 \mathrm{E}-21$ & $4,2518 \mathrm{E}-11$ & $462 \%$ \\
\hline $\mathrm{Sb}$ & 51 & 70 & 121,8 & $3,049 \mathrm{E}-02$ & B.24E-21 & $1,48 \mathrm{E}-21$ & $4,0717 \mathrm{E}-11$ & $457 \%$ \\
\hline
\end{tabular}

\begin{tabular}{|c|c|c|c|c|c|c|c|c|}
\hline ELEMENT & $\mathbf{z}$ & $\mathbf{N}$ & A & $E_{2}=E_{1}=E$ & $\sigma_{2}$ & $\sigma_{1}$ & $\lambda$ & $(02-\sigma 1) \mathrm{No1}$ \\
\hline & & & A & $\mathrm{MeV}$ & $\mathrm{cm}^{2}$ & $\mathrm{~cm}^{2}$ & m & $\%$ \\
\hline Te & 52 & 76 & 127,6 & $3,180 \mathrm{E}-02$ & $7,88 \mathrm{E}-21$ & $1,43 \mathrm{E}-21$ & $3,9041 \mathrm{E}-11$ & 452 \\
\hline 1 & 53 & 74 & 126,9 & $3,317 \mathrm{E}-02$ & $7,55 \mathrm{E}-21$ & $1,38 \mathrm{E}-21$ & $3,7430 \mathrm{E}-11$ & $447 \%$ \\
\hline$x_{e}$ & 54 & 77 & 131,3 & $3,560 \mathrm{E}-02$ & $7,24 \mathrm{E}-21$ & $1,34 \mathrm{E}-21$ & $3,4874 \mathrm{E}-11$ & $442 \%$ \\
\hline $\mathrm{Cs}$ & 55 & 78 & 132,9 & $3,590 \mathrm{E}-02$ & $6,93 \mathrm{E}-21$ & $1,29 \mathrm{E}-21$ & $3,4583 \mathrm{E}-11$ & $436 \%$ \\
\hline Ba & 56 & 81 & 137,3 & $3,740 \mathrm{E}-02$ & $6,66 \mathrm{E}-21$ & $1,25 \mathrm{E}-21$ & $3,3196 \mathrm{E}-11$ & $431 \%$ \\
\hline La & 57 & \begin{tabular}{|l|}
82 \\
\end{tabular} & 138,9 & $3,890 \mathrm{E}-02$ & $6,39 \mathrm{E}-21$ & $1,22 \mathrm{E}-21$ & $3,1916 \mathrm{E}-11$ & $426 \%$ \\
\hline $\mathrm{Ce}$ & 58 & 82 & 140,1 & $3,598 \mathrm{E}-02$ & $6,13 \mathrm{E}-21$ & $1,92 \mathrm{E}-21$ & $3,4506 \mathrm{E}-11$ & $219 \%$ \\
\hline $\mathrm{Pr}$ & 59 & 82 & 140,9 & $4,199 \mathrm{E}-02$ & $5,89 \mathrm{E}-21$ & $1,14 \mathrm{E}-21$ & $2,9567 \mathrm{E}-11$ & $415 \%$ \\
\hline $\mathrm{Nd}$ & 60 & 83 & 144,2 & $4,360 \mathrm{E}-02$ & $5,68 \mathrm{E}-21$ & $1,11 \mathrm{E}-21$ & $2,8475 \mathrm{E}-11$ & $411 \%$ \\
\hline $\mathrm{Pm}$ & 61 & 84 & 145,0 & $4,520 \mathrm{E}=02$ & $5,47 \mathrm{E}-21$ & $1,08 \mathrm{E}-21$ & $2,7467 \mathrm{E}-11$ & $06 \%$ \\
\hline $\mathrm{Sm}$ & 62 & 88 & 150,4 & $4,680 \mathrm{E}-02$ & $5,24 E-21$ & $1,05 \mathrm{E}-21$ & $2,6528 \mathrm{E}-11$ & $399 \%$ \\
\hline Eu & 63 & 88 & 152,0 & $4,850 \mathrm{E}-02$ & $5,05 \mathrm{E}-21$ & $1,02 \mathrm{E}-21$ & $2,5598 \mathrm{E}-11$ & $394 \%$ \\
\hline Gd & 64 & 93 & 157,3 & $5,023 \mathrm{E}-02$ & $4,92 \mathrm{E}-21$ & $9,95 \mathrm{E}-22$ & $2,4717 \mathrm{E}-11$ & $94 \%$ \\
\hline $\mathrm{Tb}$ & 65 & 94 & 158,9 & $5,200 \mathrm{E}-02$ & $4,69 \mathrm{E}-21$ & $9,69 \mathrm{E}-22$ & $2,3877 \mathrm{E}-11$ & $84 \%$ \\
\hline Dy & 66 & 97 & 162,5 & $5,379 \mathrm{E}-02$ & $4,52 E-21$ & $9,44 \mathrm{E}-22$ & $2,3081 \mathrm{E}-11$ & $379 \%$ \\
\hline Ho & 67 & 98 & 164,9 & $5,560 \mathrm{E}-02$ & $4,35 \mathrm{E}-21$ & $9,20 \mathrm{E}-22$ & $2,2329 \mathrm{E}-11$ & $73 \%$ \\
\hline Er & 68 & 99 & 167,3 & $5,749 \mathrm{E}-02$ & $4,21 \mathrm{E}-21$ & $8,98 \mathrm{E}-22$ & $2,1597 \mathrm{E}-11$ & $68 \%$ \\
\hline Tm & 69 & 122 & 204,4 & $5,940 \mathrm{E}-02$ & $4,07 \mathrm{E}-21$ & $1,06 \mathrm{E}-21$ & $2,0901 \mathrm{E}-11$ & $85 \%$ \\
\hline Yo & 70 & 103 & 173,1 & $6,133 \mathrm{E}-02$ & $3,92 \mathrm{E}-21$ & $8,53 \mathrm{E}-22$ & $2,0242 \mathrm{E}-11$ & $60 \%$ \\
\hline Lu & 71 & 104 & 175,0 & $6,331 \mathrm{E}-02$ & $3,79 E-21$ & $8,35 \mathrm{E}-22$ & $1,9609 \mathrm{E}-11$ & $354 \%$ \\
\hline $\mathrm{Hf}$ & 72 & 72 & 178,5 & $6,535 \mathrm{E}-02$ & $3,67 \mathrm{E}-21$ & $1,05 \mathrm{E}-21$ & $1,8998 \mathrm{E}-11$ & $248 \%$ \\
\hline $\mathrm{Ta}$ & 73 & 108 & 180,9 & $6,742 \mathrm{E}-02$ & $3,54 \mathrm{E}-21$ & $7,97 \mathrm{E}-22$ & $1,8416 \mathrm{E}-11$ & $45 \%$ \\
\hline W & 74 & 109 & 183,8 & $6,950 \mathrm{E}-02$ & $3,42 \mathrm{E}-21$ & $7,78 \mathrm{E}-22$ & 1,78 & $39 \%$ \\
\hline $\mathrm{Re}$ & 75 & 110 & 186,2 & $7,170 \mathrm{E}-02$ & $3,31 \mathrm{E}-21$ & $7,61 \mathrm{E}-22$ & $1,7315 \mathrm{E}-11$ & $335 \%$ \\
\hline$O_{8}$ & 76 & 114 & 190,2 & $7,387 \mathrm{E}-02$ & $3,21 \mathrm{E}-21$ & $7,45 \mathrm{E}-22$ & $1,6807 \mathrm{E}-11$ & $31 \%$ \\
\hline If & 77 & 114 & 192,2 & $7,610 \mathrm{E}-02$ & $3,11 \mathrm{E}-21$ & $7,28 \mathrm{E}-22$ & $1,6314 \mathrm{E}-11$ & $27 \%$ \\
\hline $\mathrm{Pt}$ & 78 & 117 & 195,1 & $7,839 \mathrm{E}-02$ & $3,01 \mathrm{E}-21$ & $7,13 \mathrm{E}-22$ & $1,5837 \mathrm{E}-11$ & $323 \%$ \\
\hline $\mathrm{Au}$ & 79 & 118 & 197,0 & $8,070 \mathrm{E}-02$ & $2,81 \mathrm{E}-21$ & $7,00 \mathrm{E}-22$ & $1,5384 \mathrm{E}-11$ & $16 \%$ \\
\hline $\mathrm{Hg}$ & 80 & 121 & 200.6 & $8.310 \mathrm{E}-02$ & $2,82 E-21$ & $6,86 \mathrm{E}-22$ & $1.4940 \mathrm{E}-11$ & $11 \%$ \\
\hline$\pi$ & 81 & 122 & 204,4 & $8,550 \mathrm{E}-02$ & $2,73 E-21$ & $6,72 \mathrm{E}-22$ & $1,4521 \mathrm{E}-11$ & $07 \%$ \\
\hline $\mathrm{PD}$ & 82 & 125 & 207.2 & $8,800 \mathrm{E}-02$ & $2,64 E \cdot 21$ & $6,57 \mathrm{E}-22$ & $1,4107 \mathrm{E}-11$ & $302 \%$ \\
\hline$B i$ & 83 & 126 & 209,0 & $9,053 \mathrm{E}-02$ & $2,56 \mathrm{E}-21$ & $6,46 \mathrm{E}-22$ & $1,3714 \mathrm{E}-11$ & $97 \%$ \\
\hline Po & 84 & 125 & 210,0 & $9,310 \mathrm{E}-02$ & $2,49 \mathrm{E}-21$ & $6,35 \mathrm{E}-22$ & $1,3335 \mathrm{E}-11$ & $292 \%$ \\
\hline At & 85 & 125 & 210,0 & $9,570 \mathrm{E}-02$ & $2,41 \mathrm{E}, 21$ & $6,21 \mathrm{E}-22$ & $1,2973 \mathrm{E}-11$ & $288 \%$ \\
\hline Rn & 86 & 136 & 222,0 & $9,840 \mathrm{E}-02$ & $2,35 \mathrm{E}-21$ & $6,08 \mathrm{E}-22$ & $1,2617 \mathrm{E}-11$ & $6 \%$ \\
\hline $\mathrm{Fr}$ & 87 & 136 & 223,0 & $1,010 \mathrm{E}-01$ & $2,26 \mathrm{E} \cdot 21$ & $5,96 \mathrm{E}-22$ & $1,2292 \mathrm{E}-11$ & $78 \%$ \\
\hline Ra & 88 & 138 & 226,0 & $1,040 \mathrm{E}-01$ & $2,19 \mathrm{E}-21$ & $5,85 \mathrm{E}-22$ & $1,1938 \mathrm{E}-11$ & $274 \%$ \\
\hline Ac & 89 & 138 & 227,0 & 1,068E-01 & $2,12 \mathrm{E}-21$ & $5,77 \mathrm{E}-22$ & $1,1629 \mathrm{E}-11$ & $67 \%$ \\
\hline Th & 90 & 142 & 232,0 & $1,096 \mathrm{E}-01$ & $2,06 \mathrm{E}-21$ & $5,66 \mathrm{E}-22$ & $1,1328 \mathrm{E}-11$ & $263 \%$ \\
\hline $\mathrm{Pa}$ & 91 & 140 & 231,0 & $1,126 \mathrm{E}-01$ & $1,99 \mathrm{E}-21$ & $5,56 \mathrm{E}-22$ & $1,1026 \mathrm{E}-11$ & $259 \%$ \\
\hline U & 92 & 146 & 238,0 & $1,160 \mathrm{E}-01$ & $1,93 \mathrm{E}-21$ & $5,45 \mathrm{E}-22$ & $1,0703 E-11$ & $254 \%$ \\
\hline
\end{tabular}

Tabla 3. Máxima variación de la sección eficaz a energías específicas o de resonancia, para los elementos presentes en aire húmedo: $\mathrm{H}_{2}, \mathrm{He}, \mathrm{O}, \mathrm{N}, \mathrm{H}_{2} \mathrm{O}, \mathrm{O}_{2}, \mathrm{~N}_{2}$.

\begin{tabular}{|c|c|c|c|c|c|c|c|}
\hline \begin{tabular}{|l|} 
ELEMENT \\
\end{tabular} & $z$ & A & $E_{r}=E_{1}=E$ & $W(E)$ & $\sigma_{t}$ & $\sigma_{1}$ & $(02-01) / 01$ \\
\hline & & & $\mathrm{eV}$ & $\mathrm{m} / \mathrm{s}$ & $\mathrm{cm}^{2}$ & $\mathrm{~cm}^{2}$ & $\%$ \\
\hline $\mathrm{H}_{2}$ & 2 & 2,02 & 100,00 & $5,930 \mathrm{E}+06$ & $1,20 \mathrm{E}-16$ & $9,69 \mathrm{E}-17$ & $24 \%$ \\
\hline $\mathrm{He}$ & 2 & 4,00 & 100,00 & $5,930 E+06$ & $4,20 \mathrm{E}-17$ & $3,58 \mathrm{E}-17$ & $17 \%$ \\
\hline 0 & 8 & 16,00 & 100,00 & $5,930 \mathrm{E}+06$ & $1,33 \mathrm{E}-16$ & $1,95 \mathrm{E}-17$ & $583 \%$ \\
\hline N & 7 & 14,01 & 100,00 & $5,930 E+06$ & $2,00 \mathrm{E}-16$ & $1,40 \mathrm{E}-16$ & $43 \%$ \\
\hline $\mathrm{H}_{2} \mathrm{O}$ & 10 & 18,02 & 100,00 & $5,930 \mathrm{E}+06$ & $3,10 \mathrm{E}-16$ & $2,01 \mathrm{E}-16$ & $55 \%$ \\
\hline $\mathrm{O}_{2}$ & 32 & 32,00 & 100,00 & $5,930 \mathrm{E}+06$ & $3,00 \mathrm{E}-16$ & $2,50 \mathrm{E}-16$ & $20 \%$ \\
\hline $\mathrm{N}_{2}$ & 14 & 28,00 & 100,00 & $5,930 \mathrm{E}+06$ & $2,75 E-16$ & $2,50 \mathrm{E}-16$ & $10 \%$ \\
\hline $\mathrm{SO}_{2}$ & & 64,07 & 100,00 & $5,930 \mathrm{E}+06$ & $7,00 \mathrm{E}-16$ & $4,00 \mathrm{E}-16$ & $75 \%$ \\
\hline $\mathrm{CH}_{4}$ & & 16,04 & 100,00 & $5,930 \mathrm{E}+06$ & $4,00 \mathrm{E}-16$ & $3,50 \mathrm{E}-16$ & $14 \%$ \\
\hline
\end{tabular}



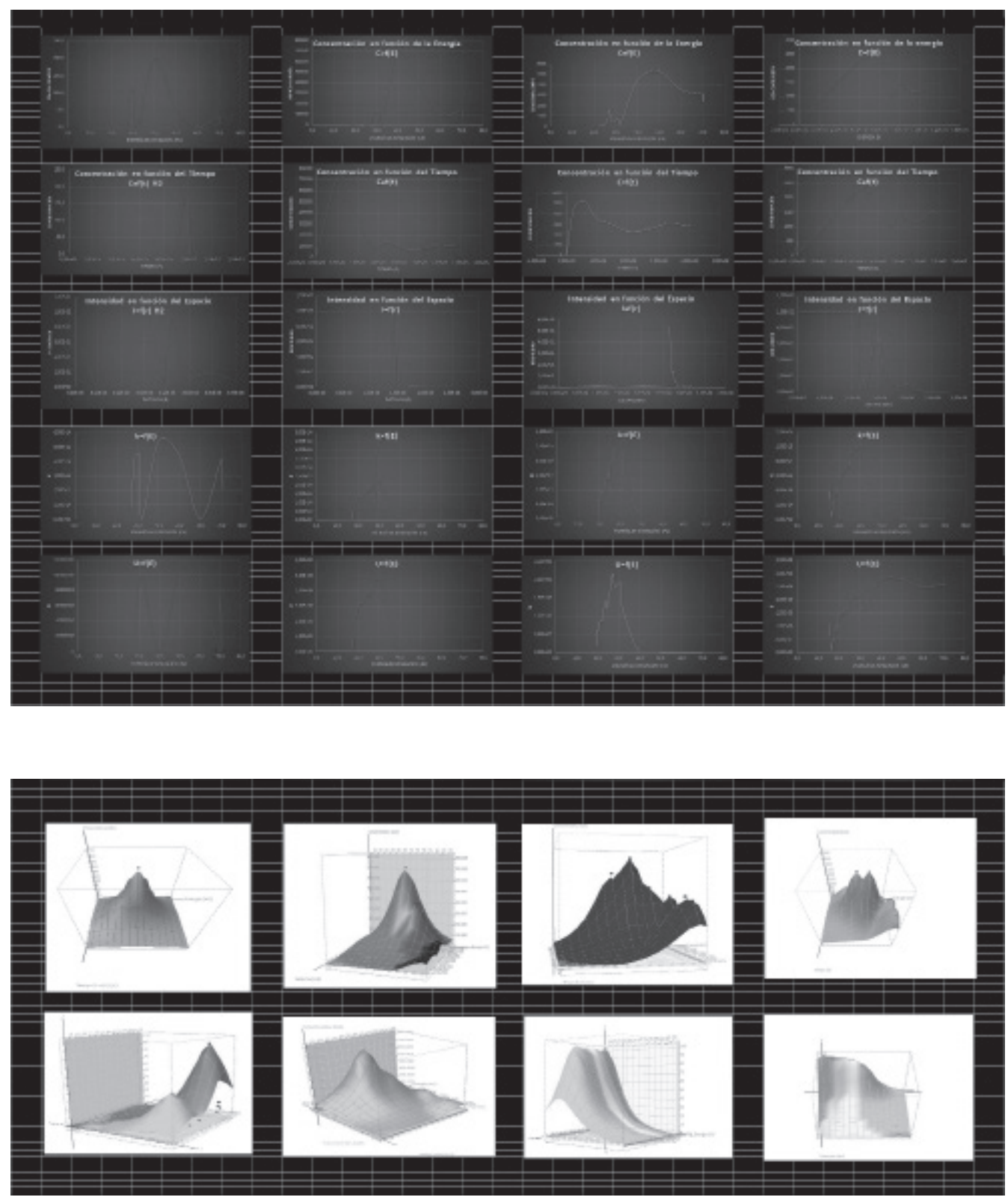

Figura 5. Evolución de concentración e intensidad de la radiación para gases de aire húmedo. 


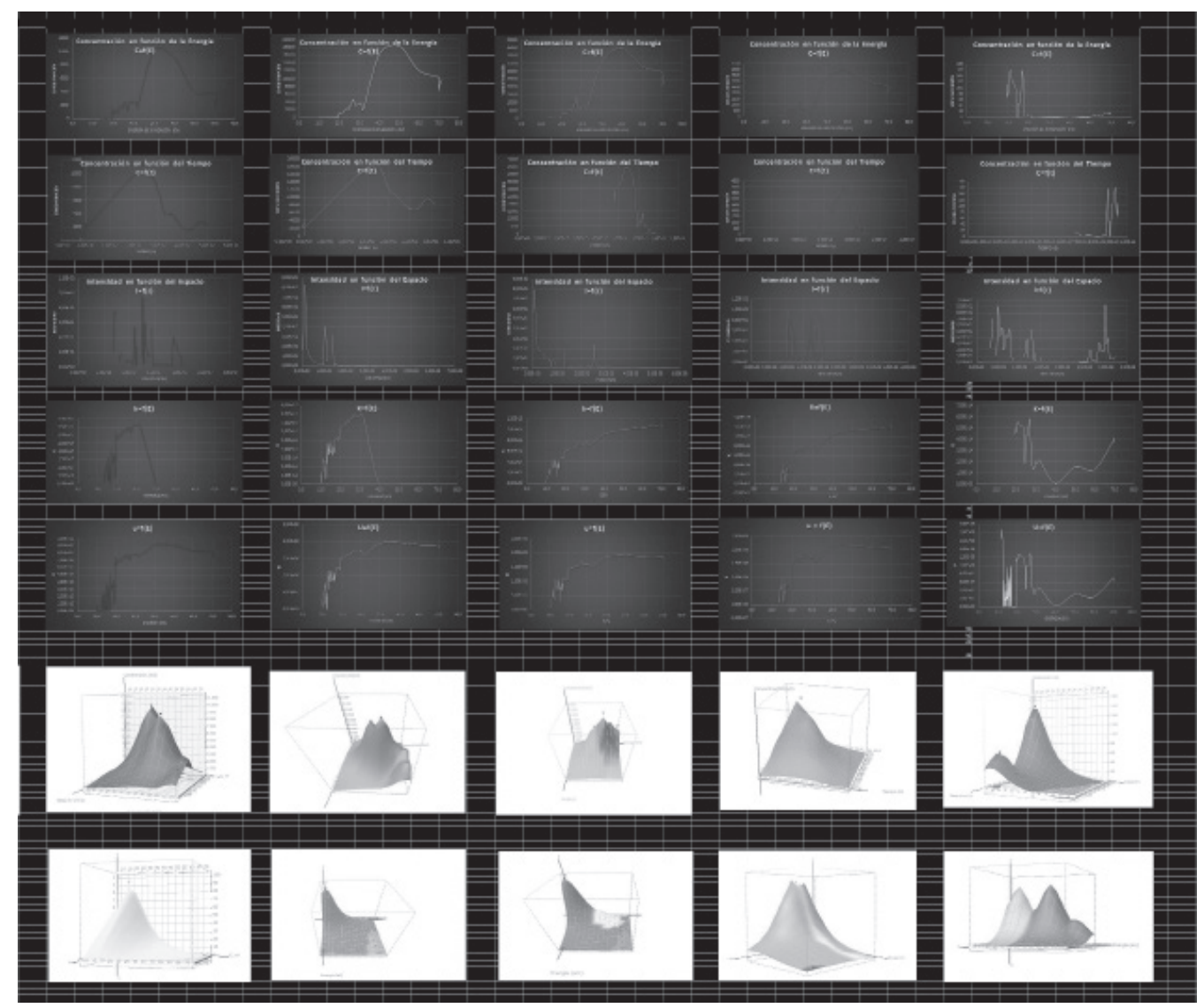

Figura 6. Evolución de concentración e intensidad de la radiación para gases de aire húmedo. Medidos en laboratorios de FIQ. 